\title{
Neutralino Annihilation at the Galactic Center Revisited
}

\author{
Roberto Aloisio $^{a}$, Pasquale Blasi ${ }^{b, c}$ and Angela V. Olinto ${ }^{d, e}$ \\ ${ }^{a}$ INFN/Laboratori Nazionali del Gran Sasso, SS 17bis, I-67010 Assergi (Italy) \\ ${ }^{b}$ INAF/Osservatorio Astrofisico di Arcetri, Largo E. Fermi 5, I-50125 Firenze (Italy) \\ ${ }^{c}$ INFN/Sezione di Firenze, Italy \\ ${ }^{d}$ Department of Astronomy \& Astrophysics, The University of Chicago, \\ 5640 S. Ellis Av., 60637 Chicago (USA) \\ ${ }^{e}$ Center for Cosmological Physics, The University of Chicago
}

\begin{abstract}
The annihilation of neutralino dark matter in the Galactic Center (GC) may result in radio signals that can be used to detect or constrain the dark matter halo density profile or dark matter particle properties. At the Galactic Center, the accretion flow onto the central Black Hole $(\mathrm{BH})$ sustains strong magnetic fields that can induce synchrotron emission by electrons and positrons generated in neutralino annihilations during advection onto the $\mathrm{BH}$. Here we reanalyze the radiative processes relevant for the neutralino annihilation signal at the GC, with realistic assumptions about the accretion flow and its magnetic properties. We find that neglecting these effects, as done in previous papers, leads to the incorrent electron and photon spectra. We find that the magnetic fields associated with the flow are significantly stronger than previously estimated. We derive the appropriate equilibrium distribution of electrons and positron and the resulting radiation, considering adiabatic compression in the accretion flow, inverse Compton scattering off synchrotron photons (synchrotron selfCompton scattering), and synchrotron self-absorption of the emitted radiation. We derive the signal for a Navarro-Frenk-White (NFW) dark matter halo profile and a NFW profile with a dark matter spike due to the central $\mathrm{BH}$. We find that the observed radio emission from the GC is inconsistent with the scenario in which a spiky distribution of neutralinos is present. We discuss several important differences between our calculations and those previously presented in the literature.
\end{abstract}

\section{Introduction}

The combination of cosmic microwave background anisotropy studies with observations of distant type Ia supernovae and measurements of the large scale structure of the universe reveal that the energy density in our universe is dominated by dark energy $(\sim 70 \%)$ followed by a significant contribution from matter $(\sim 27 \%)$. In addition, these studies show that the baryonic content of the universe is limited to less than $\sim 4 \%$ in agreement with big bang nucleosynthesis predictions. More specifically, recent WMAP results give the mean baryonic density in the universe to be within $0.044 \pm 0.004$ of the critical density while the matter density is $0.27 \pm 0.04$ of critical [1]. The remaining 
0.23 of the matter density, i.e., the bulk of the matter in the universe, is believed to be a yet undiscovered form of dark matter.

Weakly interacting massive particles (WIMPs) are natural candidates for the dark matter [2]. Particles with masses around $\sim 100 \mathrm{GeV}$ that interact only weakly have freeze-out densities in the required range. In addition, particle physics models that invoke supersymmetry generate a number of plausible WIMP candidates. In supersymmetric extensions of the standard model, the lightest supersymmetric particle may be stable due to conservation of R-parity enabling their survival to the present. In addition to massive and weakly interacting, dark matter particles are expected to be neutral. A class of neutral lightest supersymmetric particles is a combination of gauginos and higgsinos, named the neutralino often represented by $\chi$.

A number of experiments are presently searching for the neutralino (see, e.g., [3] ). Accelerator experiments have placed lower limits on the neutralino mass of $m_{\chi} \gtrsim 37$ $\mathrm{GeV}$ [4]. The neutralino mass in the minimal supersymmetric extension of the standard model that can explain the dark matter density is also bounded from above, $m_{\chi} \lesssim 7$ $\mathrm{TeV}$. In some constrained supersymmetric models $m_{\chi} \lesssim 500 \mathrm{GeV}$ due to the cosmological constraints from WMAP [5]. Direct searches for neutralinos that cross the Earth are presently reaching the required sensitivity to probe the relevant range in parameter space of supersymmetric models.

A good complement to accelerator and direct detection methods are indirect searches based on the emission from neutralino self-annihilation in astrophysical environments 6, 17, 8, 9, 10, 11, 12, 13, 14, 15, 16. Neutralinos self-annihilate at a rate proportional to the square of the neutralino density. Thus, the highest density dark matter regions are the best candidates for indirect searches. In fact, the GC region may potentially be so dense that all neutralino models would be ruled out [9]. This strong constraint arises in models where the super-massive black hole (BH) at the GC induces a strong dark matter density peak called the spike. The existence of such a spike is strongly dependent on the formation history of the Galactic Center BH [17. If the $\mathrm{BH}$ formed adiabatically a spike would be present, while a history of major mergers would most likely not allow the survival of a spike.

In contrast to the uncertain presence of a central spike in the dark matter distribution, the central $\mathrm{BH}$ is known to induce an accretion flow of baryonic matter around its event horizon. The accretion flow carries magnetic fields, possibly amplified to near equipartition values due to the strong compression. The distribution of electrons and positrons (hereafter both called electrons) produced by neutralino annihilation at the $\mathrm{GC}$ would also be compressed toward the $\mathrm{BH}$ radiating through synchrotron and inverse Compton scattering off the local photon background.

By considering the injection of electrons, combined with radiative losses and adiabatic compression, we find the equilibrium spatial and spectral electron distribution and derive the expected radiation signal. We find that the synchrotron emission of electrons from neutralino annihilation ranges from radio and microwave energies up to the optical, in the central more magnetized region of the accretion flow. At 
low frequencies, synchrotron self-absorption (SSA) reduces the amount of radiation transmitted outwards. The resulting signal is stronger than the observed emission in the 10 to $10^{5} \mathrm{GHz}$ range for the case of a spiky dark matter profile while for a pure NFW profile the emission is below the observed level. Here, we do not attempt to explore all possible spike profiles in the GC region, that might originate from the adiabatic compression of different types of initial dark matter profiles. Instead, we limit ourselves to the case of a spike arising from adiabatic compression of a NFW profile in the gravitational field of the massive $\mathrm{BH}$ at the $\mathrm{GC}$ (hereafter, the spike case.) in contrast to the pure NFW profile.

Previous calculations of the type presented here were either incomplete or had unphysical values for some of the parameters. In particular, calculations presented in [9] and [12] assume a magnetic field that does not correspond to the equipartition field for the accretion flow around the black hole. We show here that if this incorrect field is adopted, then the main processes responsible for reaching the equilibrium of relativistic electrons are synchrotron self-compton scattering (SSC) and adiabatic compression of the particles advected with the accretion flow, processes which were both ignored in previous calculations. On the other hand, if the correct magnetic field is adopted, the equilibrium distribution of the radiating electrons is determined by advection and synchrotron emission.

The paper is organized as follows: in $\$ 2$ we describe the spatial distribution of dark matter in the $\mathrm{GC}$ region. In $\$ 3$ we describe the accretion flow around the $\mathrm{BH}$ and its magnetic field. The injection of electrons and positrons from neutralino annihilation is considered in 94 . In $\$ 5$ we solve the transport equation for electronpositron pairs from neutralino annihilation in the presence of energy losses and adiabatic compression. Synchrotron self-absorption is discussed in 96 . Results of our calculations and comparison with observations are shown in $\S 7$ and comparison with previous work is made in $\$ 8$, We conclude in 99 .

\section{The spatial distribution of dark matter in the Galactic Center}

The spatial distribution of dark matter in halos is still a matter of much debate (see, e.g., [19]). Numerical simulations suggest that collisionless dark matter forms cuspy halos while some observations argue for a flat inner density profile [20]. The standard numerical dark matter halo is the NFW profile [21] which is expected to be universal. However, recent simulations have found more cuspy halos [22] as well as shallower profiles 23. At present, it is not clear that there is a universal dark matter halo profile, but the NFW case seems to represent well the range of plausible profiles. Therefore, we assume that the NFW profile describes well the dark matter in our Galaxy.

The NFW profile can be parametrized as

$$
\rho(r)=\frac{\rho_{0}}{\frac{r}{R_{c}}\left[1+\frac{r}{R_{c}}\right]^{2}} .
$$

where $R_{c}$ is the core radius and $\rho_{0}$ is a normalization constant. The two free parameters 
can be determined from the total mass of the Galaxy (that we take to be $2 \times 10^{12} M_{\odot}$ ) and the velocity dispersion at the solar location which gives $\rho_{\odot}=\rho_{D M}\left(R_{\odot}\right)=$ $6.5 \times 10^{-25} \mathrm{~g} / \mathrm{cm}^{3}$ for $R_{\odot}=8.5 \mathrm{kpc}$.

There is now growing evidence for the presence of a supermassive black hole at the Galactic Center, with mass $\sim 2 \times 10^{6} M_{\odot}$. In fact most galaxies seem to harbor central black holes with comparable or even larger masses. The presence of a $\mathrm{BH}$ can steepen the density profile of dark matter by transforming the cusp at the GC into a spike of dark matter 24]. The density profile of the spike region, where the gravitational potential is dominated by the $\mathrm{BH}$ is described by

$$
\rho_{s p}^{\prime}(r)=\alpha_{\delta}^{\delta_{s p}-\delta}\left(\frac{M}{\rho_{\odot} R_{\odot}^{3}}\right)^{(3-\delta)\left(\delta_{s p}-\delta\right)} \rho_{\odot} g(r)\left(\frac{R_{\odot}}{r}\right)^{\gamma_{s p}} .
$$

Here, $\delta$ is the slope of the density profile of dark matter in the inner region $(\delta=1$ for a NFW profile), and $\delta_{s p}=(9-2 \delta) /(4-\delta)$. The coefficients $\alpha_{\delta}$ and $g(r)$ can be calculated numerically as explained in detail in 24]. It is possible to identify a spike radius $R_{s p}$ where the spike density profile given in Eq. (2) matches the NFW dark matter profile. In other words, at $R_{s p}$ the gravitational potential is no longer dominated by the central $\mathrm{BH}$.

Neutralino annihilations affect the density profile in the spike by generating a flattening where the annihilation time becomes smaller than the age of the BH. This flattening occurs at the position

$$
\rho_{\text {core }}=\frac{m_{\chi}}{\langle\sigma v\rangle_{\text {ann }} t_{B H}}
$$

where $\langle\sigma v\rangle_{a n n}$ is the thermally averaged cross section times velocity for neutralino annihilation. Cosmological arguments give estimates of $\langle\sigma v\rangle_{\text {ann }} \lesssim 3 \times 10^{-26} \mathrm{~cm}^{3} \mathrm{~s}^{-1}$. The effect of annihilations on the spike density profile can be written as

$$
\rho_{s p}(r)=\frac{\rho_{s p}^{\prime}(r) \rho_{\text {core }}}{\rho_{s p}^{\prime}(r)+\rho_{\text {core }}},
$$

which accounts for the flattening in the central region.

Several dynamical effects may weaken or destroy the spike in the GC [2, 17, 18, depending on the history of formation of the central BH. If the spike is not formed or gets destroyed, the central region should be described by the cuspy profile as in the NFW case. Here we consider both cases and show that the observed emission is stronger than the predictions for a NFW cusp, while the spike generates a signal well above the observed data.

\section{The accretion flow and its magnetic field}

We model the accretion flow of gas onto the black hole at the center of our Galaxy following a simple approach described in [25]. More detailed models of the accretion flow around the $\mathrm{BH}$ lead to corrections which are negligible when compared to the uncertainties in the dark matter distribution. In the model we adopt, the BH accretes 
its fuel from a nearby molecular cloud, located at about $0.01 \mathrm{pc}$ from the BH. The accretion is assumed to be spherically symmetric Bondi accretion with a rate of mass accretion of $\dot{M}=10^{22} \dot{M}_{22} \mathrm{~g} \mathrm{~s}^{-1}$. The accretion onto the BH occurs with a velocity around the free-fall velocity, such that

$$
v(r)=\sqrt{2 G M_{B H} / r}=c\left(\frac{R_{g}}{r}\right)^{1 / 2}
$$

where $R_{g}=2 G M_{B H} / c^{2}=7.4 \times 10^{11}\left(M_{B H} / 2.5 \times 10^{6} M_{\odot}\right) \mathrm{cm}$ is the gravitational radius of the $\mathrm{BH}$ and $M_{B H}$ is the $\mathrm{BH}$ mass. Therefore,

$$
v(r)=1.46 \times 10^{8}\left(\frac{M_{B H}}{2.5 \times 10^{6} M_{\odot}}\right)^{1 / 2}\left(\frac{r}{0.01 \mathrm{pc}}\right)^{-1 / 2} \mathrm{~cm} \mathrm{~s}^{-1} .
$$

Mass conservation then gives the following density profile:

$$
\rho(r)=\frac{\dot{M}}{4 \pi r^{2} v(r)}=\frac{\dot{M}}{4 \pi R_{g}^{2} c}\left(\frac{r}{R_{g}}\right)^{-3 / 2}
$$

such that

$$
\rho(r)=5.6 \times 10^{-21} \dot{M}_{22}\left(\frac{M_{B H}}{2.5 \times 10^{6} M_{\odot}}\right)^{-1 / 2}\left(\frac{r}{0.01 \mathrm{pc}}\right)^{-3 / 2} \mathrm{~g} \mathrm{~cm}^{-3} .
$$

Following [25, we assume that the magnetic field in the accretion flow achieves its equipartition value with the kinetic pressure, namely $\rho v^{2} / 2=B(r)^{2} / 8 \pi$. With this assumption,

$$
B_{e q}(r)=\frac{\sqrt{\dot{M} c}}{R_{g}}\left(\frac{r}{R_{g}}\right)^{-5 / 4}=3.9 \times 10^{4} \dot{M}_{22} M_{B H}^{1 / 4}\left(\frac{r}{0.01 \mathrm{pc}}\right)^{-5 / 4} \mu \mathrm{G} .
$$

It is believed that magnetic fields in the accretion flow will in general reach the equipartition values described in Eq. (9). However, smaller fields may be reached if the equipartition is prevented somehow. In fact, previous authors [9, 12] have assumed a lower field given by

$$
B_{\text {low }}(r)=1 \mu \mathrm{G}\left(\frac{r}{1 \mathrm{pc}}\right)^{-5 / 4}=3 \times 10^{2} \mu \mathrm{G}\left(\frac{r}{0.01 \mathrm{pc}}\right)^{-5 / 4} \simeq 10^{-2} B_{\text {eq }},
$$

although this field was actually referred to as the equipartition field. In what follows, we assume the equipartition field as calculated in Eq. (9), and derive the signal from neutralino annihilation. In $₫ 8$, we address the case of assuming a lower filed such as in Eq. (101).

\section{Neutralino annihilations: the injection of electrons and positrons}

Neutralino annihilations can result in many different final states (see, e.g. [13] and references therein). Given the large uncertainties in the dark matter distribution, we chose to make the simplifying assumption that the annihilation channels are dominated by quarks and antiquarks, which in turn generate mainly pions:

$$
\chi+\chi \rightarrow q+\bar{q} \rightarrow \text { pions. }
$$


Approximately $1 / 3$ of the pions are neutral and promptly decay to gamma rays. About $2 / 3$ of the pions are charged and result in the production of electrons, positrons and neutrinos. Although much attention has been devoted in the past literature to the gamma rays from $\pi^{0}$ decays, electrons and positrons can result in copious production of nonthermal radiation in the presence of magnetic fields and a photon background.

We simplify the annihilation treatment further by assuming that the spectrum of charged pions in a single neutralino annihilation is described by the Hill [26] quark fragmentation:

$$
W_{\pi}\left(E_{\pi}\right)=\frac{5}{4} \frac{1}{m_{\chi}} x^{-3 / 2}(1-x)^{2},
$$

where $x=E_{\pi} / m_{\chi}$. In Eq. (12), a $2 / 3$ factor accounts for the fraction of charged pions compared to neutral $(1 / 3)$ in each neutralino annihilation event, and a factor 2 accounts for two jets, each with energy $m_{\chi}$, the neutralino mass. With these simplifying assumptions, the spectrum of generated electrons appears to possess the main features found with a full treatment of the neutralino annihilations (see 13. for a description of the full calculation), although the latter should be used for a more quantitative description of the particle physics aspects of the problem.

The spectrum of electrons (and positrons) from the $\pi^{ \pm}$decays is calculated by convoluting the spectrum of pions and muons. For relativistic electrons the electron spectrum reads

$$
\begin{array}{r}
W_{e}\left(E_{e}\right)=\int_{\max \left(E_{e}, m_{m u}\right)}^{m_{\chi}} d E_{\mu} \int_{E_{\pi}^{\min }\left(E_{\mu}\right)}^{E_{\pi}^{\max }\left(E_{\mu}\right)} d E_{\pi} W_{\pi}\left(E_{\pi}\right) . \\
\cdot \frac{m_{\pi}^{2}}{m_{\pi}^{2}-m_{\mu}^{2}} \frac{1}{\sqrt{E_{\pi}^{2}-m_{\pi}^{2}}} \frac{d n_{e}\left(E_{e}, E_{\mu}, E_{\pi}\right)}{d E_{e}}
\end{array}
$$

where, neglecting the muon polarization, we get

$$
\frac{d n_{e}\left(E_{e}, E_{\mu}, E_{\pi}\right)}{d E_{e}}=\frac{1}{E_{\mu} \beta}\left\{\begin{array}{lll}
2\left[\frac{5}{6}-\frac{3}{2} \epsilon^{2}+\frac{2}{3} \epsilon^{3}\right] & \text { if } & \frac{1-\beta}{1+\beta} \leq \epsilon \leq 1 \\
\frac{4 \epsilon^{2} \beta}{(1-\beta)^{2}}\left[3-\frac{2}{3} \epsilon\left(\frac{3+\beta^{2}}{1-\beta}\right)^{2}\right] & \text { if } & 0 \leq \epsilon \leq \frac{1-\beta}{1+\beta}
\end{array}\right.
$$

with $\epsilon=\frac{2}{1+\beta} \frac{E_{e}}{E_{\mu}}$. Here $\beta$ is the pion speed and $E_{e}$ and $E_{\mu}$ are the total energies of electrons and muons respectively. The two limits of integration $E_{\pi}^{\min }\left(E_{\mu}\right)$ and $E_{\pi}^{\max }\left(E_{\mu}\right)$ can be derived by inverting the following equations:

$$
\begin{aligned}
& E_{\mu} \leq \frac{E_{\pi}}{2 m_{\pi}^{2}}\left[m_{\pi}^{2}(1+\beta)+m_{\mu}^{2}(1-\beta)\right] \\
& E_{\mu} \geq \frac{E_{\pi}}{2 m_{\pi}^{2}}\left[m_{\pi}^{2}(1-\beta)+m_{\mu}^{2}(1+\beta)\right] .
\end{aligned}
$$

Finally, the injection of new electrons at the distance $r$ from the $\mathrm{BH}$ at energy $E$ can be written as follows:

$$
Q(E, r)=\frac{1}{2}\left(\frac{\rho_{D M}(r)}{m_{\chi}}\right)^{2} W_{e}(E)\langle\sigma v\rangle_{a n n}
$$


where the density of dark matter has the profile $\rho_{D M}(r)$ discussed in $\$ 2$ with or without a spike. In this equation, the factor $1 / 2$ is introduced to avoid double counting of the annihilating neutralinos, as pointed out in [16].

\section{The equilibrium distribution of electrons near the $\mathrm{BH}$}

The spectrum of particles at a position $r$ in the accretion flow around the $\mathrm{BH}$ is the result of the injection of newly produced electrons at the same position, radiative losses of these electrons, and the adiabatic compression that may enhance their momentum as they move inward. Here we neglect spatial diffusion, which occurs on larger time scales.

The transport equation including all these effects can be written as follows:

$$
v(r) \frac{\partial f}{\partial r}-\frac{1}{3 r^{2}} \frac{\partial}{\partial r}\left[r^{2} v(r)\right] p \frac{\partial f}{\partial p}+\frac{1}{p^{2}} \frac{\partial}{\partial p}\left[p^{2} \dot{p}(r, p) f\right]=Q(r, p),
$$

where $f(r, p)$ is the equilibrium distribution function of electrons injected according to $Q(r, p)$ that lose energy radiatively as described by the function $\dot{p}(r, p)=d p(r, p) / d t$. Here $v(r)=-c\left(r / R_{g}\right)^{-1 / 2}$ is the inflow velocity. The equation can be solved analytically if the electrons remain relativistic everywhere in the fluid, which is a good approximation for the range of frequencies of the radiation that we are interested in.

In Eq. (18), the term

$$
\dot{p}_{a d}=-\frac{1}{3} p \nabla v(r)=-\frac{1}{3 r^{2}} p \frac{\partial}{\partial r}\left[r^{2} v(r)\right]
$$

describes the rate of change of momentum of a particle at the position $r$ due to adiabatic compression in the accretion flow. The rate of adiabatic momentum enhancement should be compared with the rate of losses due to synchrotron emission:

$$
\dot{p}_{s y n}(r, p)=\frac{4}{3} \sigma_{T} \frac{B^{2}(r)}{8 \pi} \gamma^{2},
$$

where $\sigma_{T}$ is the Thomson cross section and $\gamma$ is the Lorentz factor of the electron. The magnetic field $B_{e q}(r)$ depends on $r$ as described in Eq. (9). Comparing the absolute values of the adiabatic compression rate and the rate of synchrotron losses, we find that the effect of adiabatic compression dominates at

$$
\frac{r}{R_{g}}>32.7 \gamma
$$

For this estimate we used the fiducial value of the $\mathrm{BH}$ mass $\left(2.5 \times 10^{6} M_{\odot}\right)$ and the accretion rate $\left(\dot{M}=10^{22} \mathrm{~g} \mathrm{~s}^{-1}\right)$. A graphical comparison of the time scales for adiabatic compression (solid lines) and synchrotron losses (dashed lines) is plotted in Fig. 1 for $\gamma=10$ and $\gamma=1000$.

It is clear from Fig. 1 that for Lorentz factors $\gamma<1000$, there is a region (large radii) for which the particles injected at $r$ are advected inward faster than they lose energy through synchrotron emission. If instead of using $B_{e q}(r)$ from Eq. (9), we adopted $B_{\text {low }}(r)$ from Eq. (10) as in [9, 12, the relation for the dominance of the adiabatic compression would become

$$
\frac{r}{R_{g}}>2.3 \times 10^{-3} \gamma .
$$




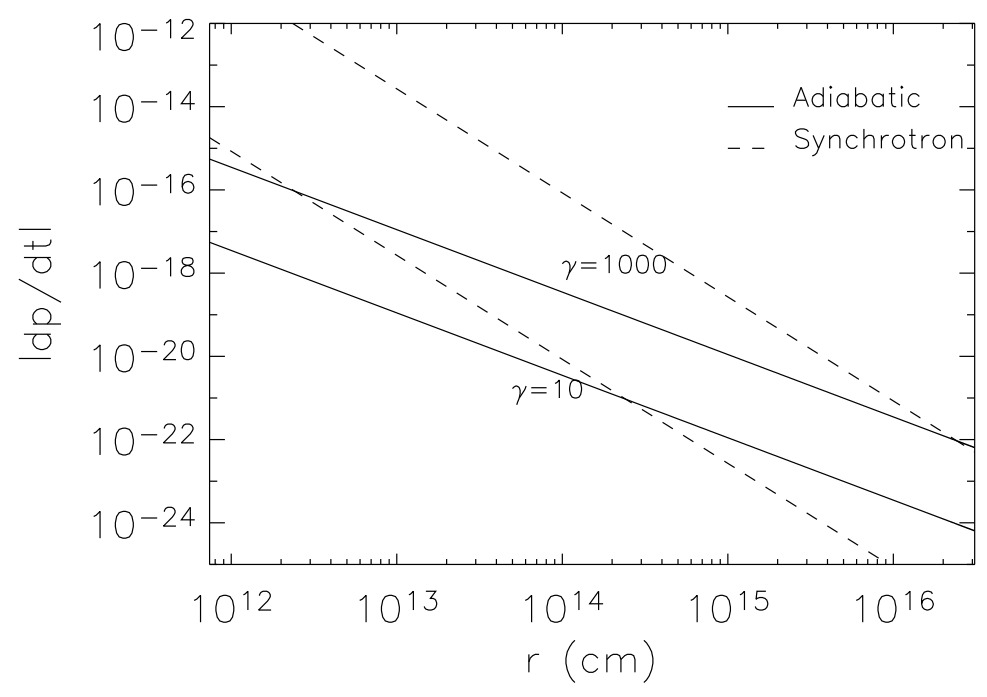

Figure 1. Comparison between the time scales for adiabatic compression (solid lines) and synchrotron losses (dashed lines) for $\gamma=10$ and $\gamma=1000$.

In this case, the adiabatic compression (ignored in previous calculations) becomes more important than synchrotron losses at most energies. The final spectrum would then differ substantially from that obtained in [9, 12, as we discuss in 88

In order to solve the transport equation, Eq. (18), we discuss different loss processes using the equipartition field $B_{e q}$. We first consider synchrotron losses $\left(\dot{p}_{s y n}\right)$, followed by inverse Compton losses $\left(\dot{p}_{I C S}\right)$ and synchrotron self-compton scattering $\left(\dot{p}_{S S C}\right)$.

The rate of synchrotron losses from Eq. (201) is given by:

$$
\dot{p}_{s y n}(r, p) c=1.6 \times 10^{-18}\left(\frac{r}{0.01 \mathrm{pc}}\right)^{-5 / 2} \gamma^{2} \operatorname{erg~s}^{-1} .
$$

In the Thomson regime, losses due to Inverse Compton Scattering (ICS) off a photon background with energy density $U_{p h}$ has the following form

$$
\dot{p}_{I C S}(r, p)=\frac{4}{3} \sigma_{T} U_{p h}(r) \gamma^{2} .
$$

ICS dominates synchrotron losses only if $B^{2}>8 \pi U_{p h}$.

Assuming that $U_{p h}$ is independent of $r$, i.e., a fixed photon background, ICS becomes important at large radii $(\sim 0.01 \mathrm{pc})$ and only if $U_{p h} \gtrsim 10^{4} \mathrm{eV} \mathrm{cm}^{-3}$. Such strong photon background is unlikely to be present at the GC region. For comparison, the CMB radiation has $U_{C M B} \approx 0.25 \mathrm{eV} \mathrm{cm}^{-3}$ while the optical background has $U_{\text {opt }} \approx 1 \mathrm{eV} \mathrm{cm}^{-3}$. If ICS off a fixed background is not dominant at large radii, it becomes even less important at small radii when compared to synchrotron losses. Consequently, we safely neglect the role of ICS off photons of fixed photon backgrounds. 
The electrons, radiating in the strong magnetic field near the BH generate a photon background that can become quite intense. The rate of losses due to ICS of electrons off the photons generated through synchrotron emission by the same electrons is

$$
\dot{p}_{S S C}(r, p)=\frac{4}{3} \sigma_{T} U_{p h}^{s y n}(r) \gamma^{2}
$$

where the photon energy density generally depends on the radius $r$. The photon density $U_{p h}^{s y n}(r)$ is a nonlinear function of the distribution $f(r, p)$. In other words, the term $\dot{p}$ in Eq. (18) depends on $f(r, p)$ when synchrotron self-Compton scattering is included. If this contribution cannot be neglected, an analytical solution of the transport equation becomes unattainable.

Given the distribution function $f(r, p)$, one can calculate the synchrotron emissivity $j(\nu, r)$ (energy per unit volume, per unit frequency, per unit time). The photon energy density at the position $r$ is then proportional to the integration over all lines of sight of the emissivity, with the possible synchrotron self-absorption taken into account at each frequency. However, the distribution function $f(r, p)$ is not known a priori, and the problem becomes intrinsically nonlinear. The approach that we follow here is to start with neglecting synchrotron self-Compton scattering and check a posteriori whether the assumption is correct in the situation at hand.

An analytic solution of the transport equation can be derived when $\dot{p}(r, p)$ is dominated by synchrotron losses as in Eq. (20). In this case, the equation admits the following analytical solution:

$$
\begin{aligned}
f(r, p)=\frac{1}{c}\left(\frac{r}{R_{g}}\right)^{-2} \int_{r}^{R_{a c c}} d R_{i n j}\left(\frac{R_{i n j}}{R_{g}}\right)^{5 / 2} \cdot \\
\cdot\left(\frac{p_{i n j}\left[p, r, R_{i n j}\right]}{p}\right)^{4} Q\left(R_{i n j}, p_{i n j}\left[p, r, R_{i n j}\right]\right) .
\end{aligned}
$$

We rewrite the synchrotron losses in the form:

$$
\dot{p}_{\text {syn }}=k_{0}\left(\frac{r}{R_{g}}\right)^{-5 / 2} p^{2}
$$

where $k_{0}=\sigma_{T} B_{0}^{2} / 6 \pi(m c)^{2}$. The function $p_{i n j}\left[p, r, R_{i n j}\right]$ corresponds to the injection momentum of an electron injected at the position $R_{i n j}$ that arrives at the position $r$ with momentum $p$. This injection momentum can be obtained by inverting, with respect to $p_{i n j}$ the solution of the equation of motion of the electron, in the presence of adiabatic compression and radiative losses:

$$
\frac{d p}{d r}=\frac{k_{0}}{c}\left(\frac{r}{R_{g}}\right)^{-2} p^{2}-\frac{1}{2 R_{g}} p\left(\frac{r}{R_{g}}\right)^{-1} .
$$

The solution of this equation, with initial condition $p\left[r=R_{i n j}, p_{i n j}, R_{i n j}\right]=p_{i n j}$ is

$$
p\left[r, p_{i n j}, R_{i n j}\right]=p_{i n j}\left[\frac{2 k_{0}}{3 c} \frac{R_{g}^{2}}{r} p_{i n j}\left[1-\left(\frac{r}{R_{i n j}}\right)^{3 / 2}\right]+\left(\frac{r}{R_{i n j}}\right)^{1 / 2}\right]^{-1} .
$$



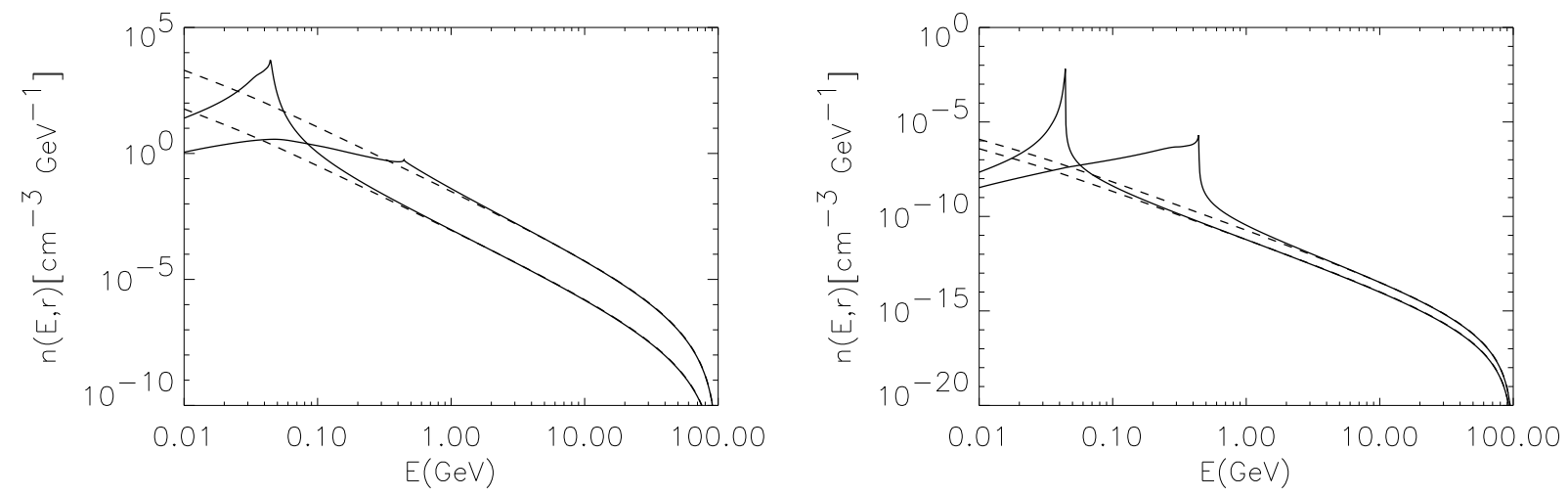

Figure 2. Electron density per unit energy as a function of energy (solid lines) at $r=10^{3} R_{g}$ (upper curve) and $r=10^{4} R_{g}$ (lower curve). Superimposed (dashed curves), we plot the function $n(E, r)$ obtained without the effect of advection. The left panel illustrates the spike case, while the right panel applies to the NFW case.

In the absence of synchrotron energy losses (when $k_{0}=0$ ), particle momenta only change due to adiabatic compression, and the momentum of a particle changes according with the well known $p=p_{i n j}\left(r / R_{i n j}\right)^{-1 / 2}$, valid for the case of free fall.

The joint effect of the energy gain due to the adiabatic compression and the energy losses due to synchrotron emission generates a new energy scale in the system. Introducing

$$
p_{m}=\frac{3 r c}{2 k_{0} R_{g}^{2}}=\frac{9 \pi r(m c)^{2}}{\sigma_{T} \dot{M}},
$$

we can rewrite $p\left[r, p_{i n j}, R_{i n j}\right]$ as:

$$
p\left[r, p_{i n j}, R_{i n j}\right]=p_{i n j}\left[\frac{p_{i n j}}{p_{m}}\left(1-\left(\frac{r}{R_{i n j}}\right)^{3 / 2}\right)+\left(\frac{r}{R_{i n j}}\right)^{1 / 2}\right]^{-1} .
$$

From this expression it is clear that, at any fixed position $r$, adiabatic compression dominates over synchrotron losses if the injection momentum is appreciably lower than $p_{m}$. In this case, the electron energy increases while the electron moves inward, until the rate of synchrotron losses becomes important. The opposite happens when the electrons are injected at momenta larger than $p_{m}$, since synchrotron losses are important from the time of injection.

The momentum $p_{m}$ can be interpreted as the momentum where the two competing effects of adiabatic heating and synchrotron losses balance each other. Thus, particles accumulate at momentum $p_{m}$. This phenomenon depends on the distance from the GC: at large distances from the $\mathrm{BH}$ the momentum $p_{m}$, which scales linearly with radius, is large and the local rate of injected electrons is low, therefore, the accumulation is small. At small distances the accumulations at $p_{m}$ becomes more evident.

We define now the equilibrium electron spectrum $n(E, r)$ at the position $r$, which 
is related to $f(E, r)$ through the relation

$$
n(E, r) d E=4 \pi p^{2} f(p, r) d p .
$$

In Fig. 2 we plot $n(E, r)$ as a function of the electron energy at two different radii, $r=10^{3} R_{g}$ (upper solid curve) and $r=10^{4} R_{g}$ (lower solid curve). The dashed lines illustrate the solution of the transport equation when adiabatic compression is switched off and only synchrotron losses are included (as in [12]). The dark matter density profile is assumed to be characterized by a spike at the center [24] in the left panel of Fig. 2. and by an NFW density profile [21] in the right panel of Fig. 2, The neutralino mass assumed in Fig. 2] was $100 \mathrm{GeV}$, while the annihilation cross-section was chosen to be $10^{-27} \mathrm{~cm}^{3} \mathrm{~s}^{-1}$. The accumulation effect described above manifests itself through the appearance of the spiky feature at the momentum $p_{m}$. The accumulation is less pronounced at large radii, as expected.

We conclude this section by addressing the issue of the synchrotron self- Compton scattering. As explained above, this effect cannot be accounted for in an analytical approach to the transport equation, since it is intrinsically nonlinear. Instead, we check a posteriori if neglecting SSC was a good assumption. Given the symmetry of the BH region, the photon energy density as a function of $r$ can be written as:

$$
U_{p h}^{s y n}(r)=\frac{1}{c}\left[\frac{1}{r^{2}} \int_{r_{\text {min }}}^{r} d r^{\prime} r^{\prime 2} \int d \nu j\left(\nu, r^{\prime}\right)+\int_{r}^{\infty} d r^{\prime} \int d \nu j\left(\nu, r^{\prime}\right)\right],
$$

where $j(\nu, r)$ is the synchrotron emissivity.

This energy density can now be compared with the magnetic energy density at the same location, $B^{2}(r) / 8 \pi$. The results are plotted in Fig. 3, the solid line represents the photon energy density $U_{p h}^{s y n}(r)$, while the magnetic energy density is plotted as a dashed line. The calculations are carried out for a dark matter density profile with the spike at the center and a neutralino mass of $100 \mathrm{GeV}$. The magnetic energy always dominates over the photon energy, although at large distances from the BH the separation between the two curves reduces to about one order of magnitude. The curves in Fig. 3 are obtained without taking into account the synchrotron self-absorption (see next section) at low frequencies, therefore the calculated photon energy density should be considered as an upper limit. On the basis of this argument, we can conclude that neglecting SSC was a reasonable approximation for the scenario at hand.

\section{Synchrotron Self-Absorption}

Synchrotron radiation can be reabsorbed by the radiating electrons when the system is sufficiently compact. This phenomenon of synchrotron self-absorption (SSA) is particularly effective at low frequencies. The radiation transfer across the GC region can be described by [27]:

$$
\frac{d I(\nu, s)}{d s}=-\chi(\nu, s) I(\nu, s)+\frac{1}{4 \pi} j(\nu, s),
$$




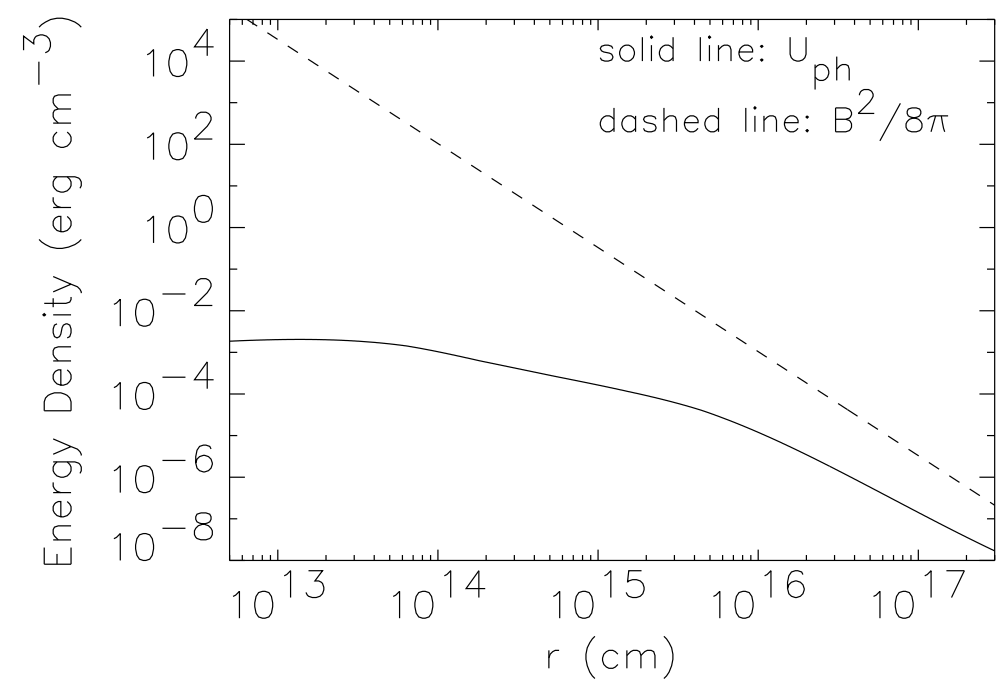

Figure 3. Comparison between the energy density of the synchrotron emitted photons $U_{p h}$ (solid line) and the energy density associated to the magnetic field $B^{2} / 8 \pi$ (dashed line).

where $I(\nu, s)$ is the radiation intensity at frequency $\nu$ along a fixed line of sight, $s$ is the linear coordinate along the line of sight, $\chi(\nu, s)$ is the absorption coefficient and $j(\nu, s)$ is the emissivity.

For sufficiently high frequencies, where the absorption coefficient is small, the radiation intensity is simply

$$
I(\nu, s)=\frac{1}{4 \pi} \int d s j(\nu, s)
$$

The absorption coefficient can be written in the form:

$$
\chi(\nu, s)=-\frac{c^{2}}{8 \pi \nu^{2}} \int d E P(\nu, E) E^{2} \frac{\partial}{\partial E}\left[\frac{n(E, r(s))}{E^{2}}\right]
$$

where $P(\nu, E)$ is the synchrotron power radiated per unit frequency by one electron of energy $E$, and $n(E, r(s))$ is the electron density per unit energy discussed in the previous section, evaluated at the position $r(s)$. Integrating equation (31) by parts, using the vanishing electron density at $E=m_{\chi}$ we obtain:

$$
\chi(\nu, s)=\frac{c^{2}}{8 \pi \nu^{2}} \int d E \frac{n(E, r)}{E^{2}} \frac{\partial}{\partial E}\left[E^{2} P(\nu, E)\right],
$$

where

$$
P(\nu, E)=\frac{\sqrt{3} e^{3} B(r)}{m c^{2}}\left(\frac{\nu}{\nu_{c}(E)}\right) \int_{\left(\frac{\nu}{\nu_{c}(E)}\right)}^{\infty} d y K_{5 / 3}(y) .
$$

Here $K_{5 / 3}$ is the modified Bessel function of order $5 / 3$ and

$$
\nu_{c}(E)=\left(\frac{3 e B(r)}{4 \pi m c}\right)\left(\frac{E}{m c^{2}}\right)^{2} .
$$




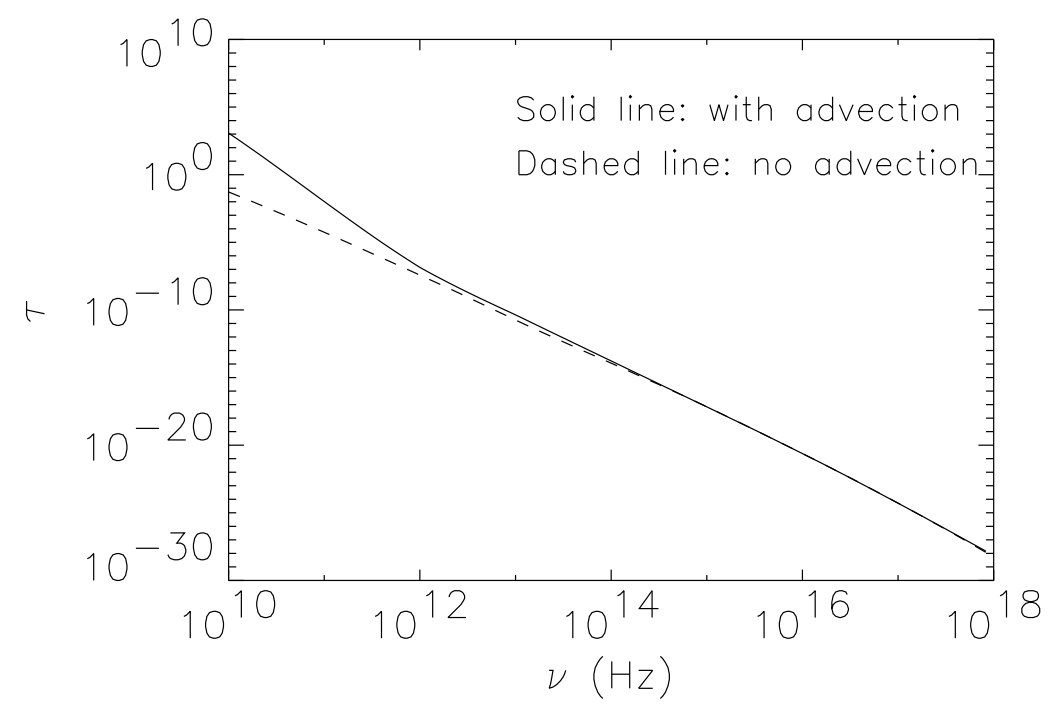

Figure 4. Optical depth as a function of the photon frequency in two cases: 1) advection plus synchrotron losses (solid line); 2) synchrotron losses only (dashed line). The calculation was carried out for a line of sight tangent to the event horizon of the black hole.

The absorption coefficient is therefore

$$
\chi(\nu, s)=\frac{c^{2}}{4 \pi} \frac{\sqrt{3} e^{3} B(r)}{m c^{2}} \int d E \frac{n(E, r)}{E \nu_{c}^{2}(E)} K_{5 / 3}\left(\frac{\nu}{\nu_{c}(E)}\right) .
$$

The effect of the SSA can be assessed by evaluating the opacity to the absorption process at fixed frequency $\nu$, defined as

$$
\tau_{\nu}(b)=\int d s \chi[\nu, r(b, s)]
$$

where $b$ is the distance of the line of sight from the line crossing the GC.

Clearly SSA becomes important when $\tau_{\nu}(b)$ approaches or exceeds unity. When $\tau_{\nu}(b) \ll 1$, SSA can be neglected and the radiation intensity can be simply evaluated as in Eq. (30). Whether the SSA is important or not depends also on the line of sight considered: in general the GC can become opaque to SSA at different frequencies for different lines of sight. The observed radiation is then the result of a line of sight integration of the radiation intensity, as described below.

In Fig. 4 we plot the opacity $\tau_{\nu}$ for a line of sight tangent to the event horizon of the BH (solid line), namely with $b=R_{g}$. In this figure, we chose the dark matter density with a spike, $m_{\chi}=100 \mathrm{GeV}$ and $\langle\sigma v\rangle_{a n n}=10^{-27} \mathrm{~cm}^{3} / \mathrm{s}$. The dashed line in Fig. 4 represents the opacity to SSA if only synchrotron losses are included while adiabatic compression is neglected (as in [12]). For the correct calculation in which both synchrotron losses and advective compression are included, the SSA becomes important for frequencies $10^{10}-10^{11} \mathrm{~Hz}$. When only synchrotron losses are considered, SSA becomes important only at much lower frequencies. 

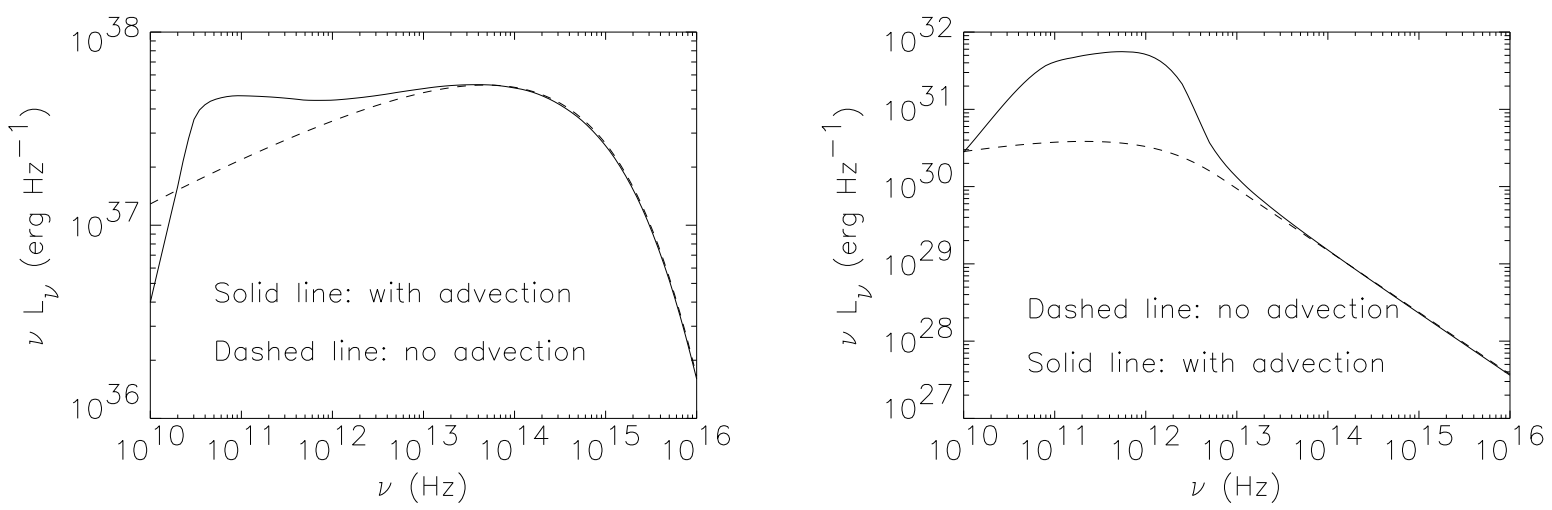

Figure 5. Emitted luminosity in two cases: 1) advection and synchrotron losses (solid line); 2) only synchrotron losses (dashed line). The computation is performed with $m_{\chi}=100 \mathrm{GeV}$ and $\langle\sigma v\rangle_{a n n}=10^{-27} \mathrm{~cm}^{3} / \mathrm{s}$. The left panel represents the Spike case while right panel the NFW case.

The dependence of the SSA on neutralino parameters scales with $n(E, r) \propto$ $\langle\sigma v\rangle_{a n n} m_{\chi}^{-3 / 2}$. At fixed $\langle\sigma v\rangle_{a n n}$, the electron density drops when $m_{\chi}$ increases, and SSA decreases as well. Finally, in the case of an NFW density profile without a spike, the rate of electron injection remains such that the effect of SSA can always be neglected at the frequencies of interest.

\section{Results}

In this section, we present our results in terms of the synchrotron luminosity produced by relativistic electrons from neutralino annihilations in the GC. We considered both cases of a spiky density profile and a NFW density profile. In order to compute the radiation intensity $I_{\nu}$, we solved Eq. (29) in the case of the spiky density profile in which, as discussed in the previous section, the SSA effect is relevant. In the case of the NFW density profile we simply used equation (30). Integrating the radiation intensity $I_{\nu}$ over the sky (namely, over all lines of sight to the observer), one obtains the luminosity:

$$
L_{\nu}=4 \pi \int d b 2 \pi b d b I_{\nu}(b) .
$$

In Fig. [5, we fix $m_{\chi}=100 \mathrm{GeV}$ and $\langle\sigma v\rangle_{\text {ann }}=10^{-27} \mathrm{~cm}^{3} / \mathrm{s}$, and show the luminosity for the spiky case (left panel) and the NFW density profile (right panel). The continuous line shows the case in which advection is taken into account, while the dashed line shows the case in which only the synchrotron energy losses are accounted for.

A comparison of the dashed and continuous curves in Fig. 5 shows that the combined effect of advection and synchrotron losses appears at frequencies up to $10^{14} \mathrm{~Hz}$. In this frequency range, the electron density is much higher when advection is included as compared to the pure synchrotron case. This effect produces an enhancement of the emitted luminosity of about one order of magnitude. In addition, we can see that 

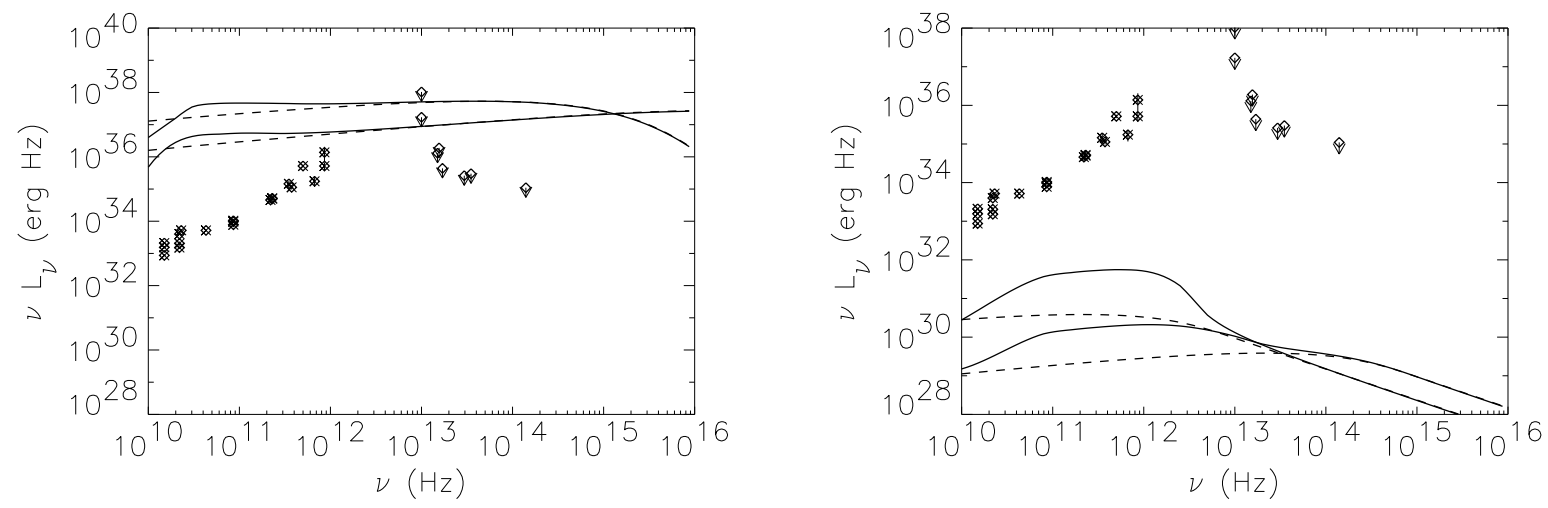

Figure 6. Luminosity, compared with experimental data from $\mathrm{Sgr} \mathrm{A}^{*}$, in the case of the spiky density profile (left panel) and NFW density profile (right panel). Dashed curves represent the luminosity obtained neglecting the effect of advection. The computation is performed with $m_{\chi}=100 \mathrm{GeV}$ (upper curves) and $m_{\chi}=1 \mathrm{TeV}$ (lower curves). The annihilation cross section is $\langle\sigma v\rangle_{\text {ann }}=10^{-27} \mathrm{~cm}^{3} / \mathrm{s}$.

SSA decreases the emitted radiation in the frequency range $10^{10}-10^{11} \mathrm{~Hz}$. This effect becomes relevant only when advection is taken into account.

In order to constrain the shape of the dark matter density profile at the GC or the parameters of the dark matter particle, we compare the calculated luminosities with the observations. The well-studied source at the GC, Sgr A*, has been observed in a large range of frequencies, from the radio to the near-infrared. Data from $10^{9} \mathrm{~Hz}$ to $10^{14} \mathrm{~Hz}$ [28 are displayed in Fig. 6. In the same figure we show the two cases of spiky (left panel) and NFW (right panel) density profiles, for $m_{\chi}=100 \mathrm{GeV}$ (upper curves) and $m_{\chi}=1 \mathrm{TeV}$ (lower curves) and $\langle\sigma v\rangle_{a n n}=10^{-27} \mathrm{~cm}^{3} / \mathrm{s}$. In Fig. [6 we also show the results for the cases in which only synchrotron losses are taken into account (dashed curves).

From Fig. 6, it is clear that a spike in the GC induces much stronger emission than the observed flux. Therefore, either there is no spike in the dark matter profile or neutralinos are not the dark matter. This conclusion qualitatively confirms what found in previous studies [9], although the magnetic field and radiative transfer here are significantly different from what was assumed in [9], which results in numerically different conclusions (see next section for a more detailed discussion of the comparison of our results with those obtained in previous literature).

The parameter space for neutralinos $\left(m_{\chi},\langle\sigma v\rangle_{a n n}\right)$ has been studied extensively [3. 29]. From accelerator and cosmological constraints $m_{\chi} \in[37 \mathrm{GeV}, 7 \mathrm{TeV}]$ and $\langle\sigma v\rangle_{a n n} \lesssim 3 \times 10^{-26} \mathrm{~cm}^{3} / \mathrm{s}$. Since the luminosity scales with the neutralino mass and the annihilation cross section through the electron density, and $n(E, r) \propto\langle\sigma v\rangle_{\text {ann }} m_{\chi}^{-3 / 2}$, the luminosity we calculated can be lowered by more than two orders of magnitude when $m_{\chi}$ is increased from $100 \mathrm{GeV}$ to $7 \mathrm{TeV}$. The luminosity increases linearly for a fixed $m_{\chi}$ as $\langle\sigma v\rangle_{a n n}$ increases. A factor of 30 increase is within the allowed parameters 
as $\langle\sigma v\rangle_{a n n}$ is changed from $10^{-27} \mathrm{~cm}^{3} / \mathrm{s}$ to $3 \times 10^{-26} \mathrm{~cm}^{3} / \mathrm{s}$. Exploring the parameter space can change our results from the fiducial choices $\left(100 \mathrm{GeV}, 10^{-27} \mathrm{~cm}^{3} / \mathrm{s}\right)$ by two orders of magnitude in either direction.

The large enhancement of the neutralino density due to the spiky profile produces a synchrotron luminosity that is difficult to reconcile with observations. Moreover, this conclusion only gets stronger when advection is included. The SSA effect does not affect the discrepancy between a neutralino spike and the observations, since it changes the luminosity only at frequencies in the range $10^{10}-10^{11} \mathrm{~Hz}$.

The situation is completely different for the case of the NFW density profile. In this case, the synchrotron luminosity is always less than the experimental data as can be seen in Fig. 6. NFW is consistent with observations even if $\langle\sigma v\rangle_{a n n}=3 \times 10^{-26}$ $\mathrm{cm}^{3} / \mathrm{s}$ is considered.

In this paper, we only considered two possibilities for the dark matter density profile: a less concentrated hypothesis (NFW) and an extremely concentrated central region (spike). As we stressed above, the spike we considered results from the adiabatic compression of a NFW dark matter profile in the gravitational field of the central BH. Less extreme spikes can be generated if the dark matter is initially distributed in a shallower manner compared with the NFW profile. On the other hand, there are cuspier

original profiles, such as the Moore et al. profile (which scales as $r^{-3 / 2}$ ). A simple Moore profile should generate a central luminosity in between the ones illustrated in this paper, while a central $\mathrm{BH}$ in such a profile will result in more pronounced spike. In some of these alternative cases, observations are likely to place more stringent limits on the neutralino parameters.

\section{Comparison with previous results}

In this section, we reconsider the case of a spike with magnetic fields as in [9, 12. We do this to show that these previous calculations have been carried out neglecting important pieces of physics, that have now been introduced. We recall that the magnetic field assumed in 9, 12 is much smaller than the real equipartition field defined in our Eq. (9), although it was quoted in these previous papers as the equipartition field. This lower field could in principle be obtained assuming considerably lower accretion rate than is usually done.

The results of [9, 12] at high frequency are obtained ignoring the effect of advection and taking only synchrotron emission as the main channel for energy losses of the electrons with a magnetic field as in Eq. (10). However, as we already discussed in previous sections, for such low fields, the effect of advection compared with synchrotron energy losses becomes very important, as follows from Eq. (201): ignoring the contribution of advection results in the incorrect prediction for the luminosity of the generated radiations.

Another important point in the comparison with the results of [9, 12] is related to the role of Synchrotron Self-Compton scattering, which was also neglected in [9, 12]. 


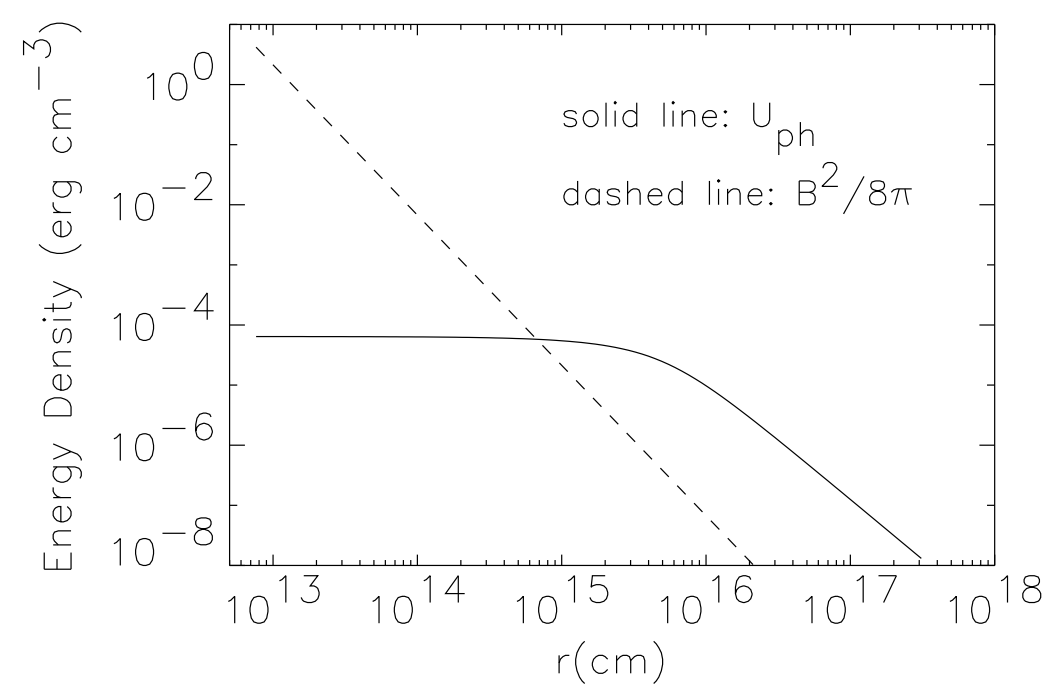

Figure 7. Comparison between the energy density of the synchrotron emitted photons $U_{p h}$ (solid line) and the energy density associated to the magnetic field $B^{2} / 8 \pi$ (dashed line), obtained fixing $B_{0}=1 \mu \mathrm{G}$ and ignoring the effect of advection (as in [9, 12]).

While SSC can indeed be neglected when using the correct equipartition magnetic field, as discussed in section 5 (see Fig. 3), this is not true in the case of a lower magnetic field (cfr. Eq. (10)) as the one used in 9, 12. In this latter case, SSC plays a crucial role. This can be easily shown by comparing the energy density of synchrotron photons $U_{p h}(r)$ with the magnetic energy density $B_{\text {low }}^{2} / 8 \pi$. The result is shown in Fig. [7 obtained adopting $m_{\chi}=10^{2} \mathrm{GeV}$ and $\langle\sigma v\rangle_{a n n}=10^{-27} \mathrm{~cm}^{3} / \mathrm{s}$. From this figure it is evident that the effect of SSC is not negligible at distances larger than $\sim 10^{3} R_{g}$ away from the GC. The effect of SSC is to lower the electron density outside about $10^{2} R_{g}$ from the GC.

We confirm that the spectrum of synchrotron radiation is affected by strong synchrotron self-absorption at the low frequency end, as also pointed out in [12. This is an important point since in [9] SSA was not included, which led to an overestimate of the low frequency synchrotron luminosity.

In Fig. 8 we neglect advection and compare observations with the emission as predicted including SSC, for $m_{\chi}=10^{2} \mathrm{GeV}$ and $\langle\sigma v\rangle_{a n n}=10^{-27} \mathrm{~cm}^{3} / \mathrm{s}$. The effect of $\mathrm{SSC}$ is not enough to reconcile the predicted luminosity with observations. The spike hypothesis remains inconsistent with observations.

\section{Conclusions}

In conclusion, we reaffirm that a spike density profile obtained from the adiabatic compression of a NFW profile in the gravitational field of the BH at the GC, using dark matter parameters typical of neutralinos, is ruled out by the radio and NIR observations from $\mathrm{SgA}^{*}$ while the NFW density profile remains a suitable hypothesis, not strongly 


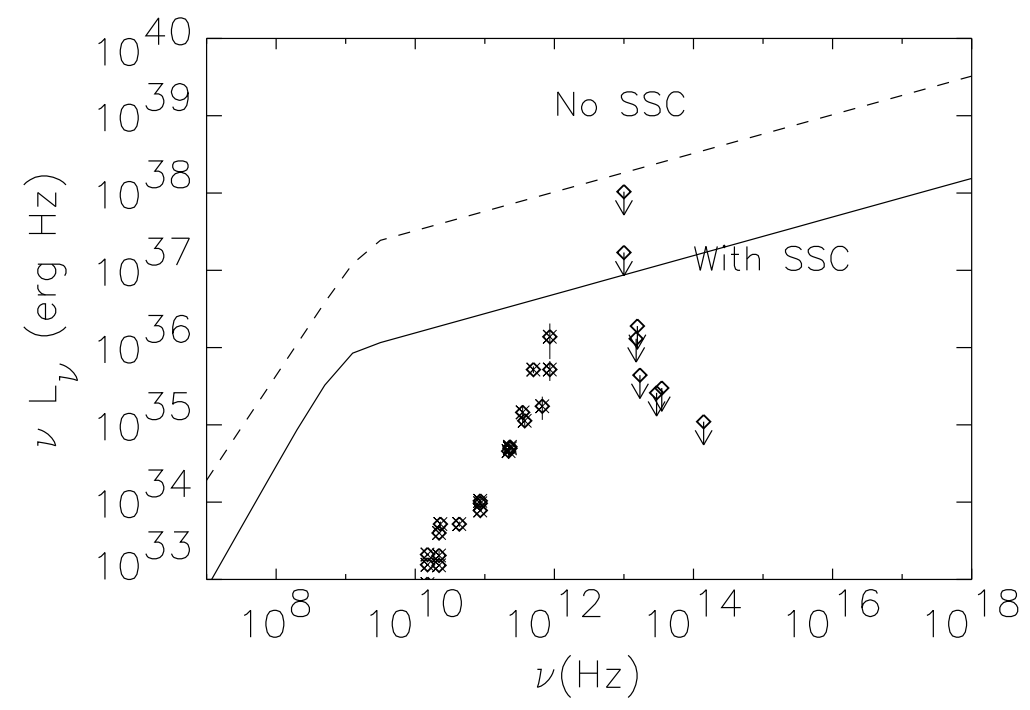

Figure 8. Luminosity obtained ignoring the effect of advection and taking into account the effect of SSC (continuos line) or ignoring it (dashed line), compared with experimental data from $\mathrm{Sgr} \mathrm{A}^{*}$, in the case of the spike density profile with $B_{0}=1$ $\mu \mathrm{G}$.

constrained by available observations.

We reached this conclusion by a careful consideration of the accretion flow and the loss processes in the transport equations for the neutralino generated electrons and positrons as well as the radiative transfer. At the Galactic Center, the accretion flow onto the central $\mathrm{BH}$ sustains strong magnetic fields that induce synchrotron emission by electrons and positrons generated in neutralino annihilations during advection onto the $\mathrm{BH}$. We found that the magnetic fields associated with the flow are significantly stronger than previously adopted. With these equipartition fields, we derive the appropriate equilibrium distribution of electrons and positrons and the resulting radiation considering adiabatic compression in the accretion flow, inverse Compton scattering off synchrotron photons (synchrotron self-Compton scattering), and synchrotron self-absorption of the emitted radiation. We calculate the signal for a NFW dark matter halo profile and a NFW profile with a dark matter spike due to the central BH. We found that the annihilation of neutralino dark matter in the GC results in radio signals that overwhelm observations if the spike is present in the dark matter density profile. However, observed emissions from the GC are consistent with neutralinos following a NFW profile at the Galactic Center.

\section{Acknowledgments}

We are grateful to Claudia Isola for a critical reading of the manuscript. The work of PB was partially funded through Cofin-2002/2003. The work of AO was supported in 
part by the NSF through grant AST-0071235 and DOEgrant DE-FG0291-ER40606 at the University of Chicago and at the Center for Cosmological Physics by grant NSF PHY-0114422.

\section{References}

[1] C.L. Bennett et al., ApJS 148, 1, 2003.

[2] G. Jungman, M. Kamionkowski, and K. Griest, Phys. Rep. 267, 195, 1996.

[3] L. Bergström, Rept. Prog. Phys. 63, 793, 2000.

[4] K. Hagiwara et al. (Particle Data Group), Phys. Rev. D66 010001, 2002.

[5] J. Ellis, K.A. Olive, Y. Santoso, V.C. Spanos, preprint hep-ph/0310356

[6] V. Berezinsky, A.V. Gurevich, and K.P. Zybin, Phys. Lett. B 294, 221, 1992.

[7] V. Berezinsky, A. Bottino and G. Mignola, Phys. Lett. B 325, 136, 1994.

[8] L. Bergström, J. Edsjö, P. Gondolo, and P. Ullio, Phys. Rev. D59, 043506, 1999.

[9] P. Gondolo, Phys. Lett. B494 181, 2000.

[10] C. Calcáneo-Roldán and B. Moore, Phys. Rev. D62, 123005, 2000.

[11] L. Bergström, J. Edsjö and P. Ullio, Phys. Rev. Lett. 87, 251301, 2001.

[12] G. Bertone, G. Sigl and J. Silk, MNRAS 326, 799, 2001.

[13] P. Ullio, L. Bergström, J. Edsjö and C. Lacey, Phys.Rev. D66, 123502, 2002.

[14] P. Blasi, A.V. Olinto and C. Tyler, Astropart. Phys. 18, 649, 2003.

[15] A. Tasitsiomi and A. V. Olinto, Phys.Rev. D66, 083006, 2002.

[16] A. Cesarini, F. Fucito, A. Lionetto, A. Morselli and P. Ullio, preprint astro-ph/0305075

[17] D. Merritt and M. Milosavljevic, in DARK 2002: 4th International Heidelberg Conference on Dark Matter in Astro and Particle Physics, 4-9 Feb 2002, Cape Town, South Africa, H.V. KlapdorKleingrothaus, R. Viollier (eds.), preprint astro-ph/0205140.

[18] P. Ullio, H.Zhao, M. Kamionkowski, Phys.Rev. D64, 043504, 2001.

[19] A. Tasitsiomi, preprint astro-ph/0205464

[20] P. Salucci, MNRAS 320, L1-L5 (2001).

[21] J.F. Navarro, C.S. Frenk and S.D.M. White, Ap. J. 462, 563, 1996; ibid. 490, 493, 1997.

[22] B. Moore, S. Ghigna, F. Governato, G. Lake, T. Quinn, J. Stadel and P. Tozzi, Astrophys.J. 524 (1999) L19.

[23] C. Power, J.F. Navarro, A. Jenkins, C.S. Frenk, S.D.M. White, V. Springel, J. Stadel, and T. Quinn, MNRAS 338 14, 2003.

[24] P. Gondolo and J. Silk, Phys. Rev. Lett. 83, 1719, 1999.

[25] F. Melia, Ap. J. 387, L25-L28, 1992.

[26] C.T. Hill, Nucl. Phys. B224, 469 (1983).

[27] G.B. Rybicki and A.P. Lightman, "Radiative Processes in Astrophysics", 1979.

[28] R. Narayan, R. Mahadevan, J.E. Grindlay, R.G. Popham and C. Gammie, Ap. J. 492, 554, 1998.

[29] T. Baltz, L. Bergström, J. Edsiö, P. Gondolo and P. Ullio, Dark SUSY review (2002), www.physto.se/ edsjo/darksusy. 